\title{
Low AC loss inkjet-printed multifilamentary YBCO coated conductors
}

\author{
Simon C. Hopkins, Tom B. Mitchell-Williams, Dries R. Vanden Bussche, Albert Calleja, Valentina Roxana Vlad, \\ Marta Vilardell, Xavier Granados, Teresa Puig, Xavier Obradors, Alexander Usoskin, Mykola Soloviov, \\ Michal Vojenčiak, Fedor Gömöry, Isabel Van Driessche, Michael Bäcker, Bartek A Glowacki
}

\begin{abstract}
Impressive performance has been achieved in $(\mathrm{RE}) \mathrm{Ba}_{2} \mathrm{Cu}_{3} \mathrm{O}_{7-\delta}$ ( $\left.\mathrm{REBCO}\right)$ coated conductors, but for many applications, the high cost and $\mathrm{AC}$ losses remain prohibitive. Inkjet printing methods combine scalability and low equipment cost with high resolution patterning, potentially addressing both issues by enabling the production of multifilamentary coated conductors without subtractive processing. The successful production of multifilamentary superconducting $\mathrm{YBa}_{2} \mathrm{Cu}_{3} \mathrm{O}_{7-\delta}$ (YBCO) structures by inkjet printing of a low-fluorine YBCO precursor solution on SS/ABAD-YSZ/CZO substrates is reported. Two approaches have been developed. In the first method, YBCO filaments were deposited directly on the buffered substrate by piezoelectric printing; and in the second approach, $\mathrm{CeO}_{2}$ tracks were first printed to pattern a subsequently overprinted YBCO film, creating a multifilamentary structure by an inverse technique. Scanning Hall probe measurements have been used to compare the filamentary structures and critical currents achieved by both methods, and a $J_{c}$ of up to $3 \mathrm{MA} \mathrm{cm}^{-2}$ has been obtained at $77 \mathrm{~K}$. For the inverse printing approach, the $\mathrm{AC}$ hysteresis losses have also been measured and compared with theoretical models.
\end{abstract}

Index Terms - AC losses, digital printing, high-temperature superconductors, multifilamentary superconductors, yttrium barium copper oxide.

The research leading to these results has received funding from the European Union's Seventh Framework Programme (FP7/2007-2013) under grant agreement NMP-LA-2012-280432 (EUROTAPES)

S. C. Hopkins and T. B. Mitchell-Williams are with the Department of Materials Science and Metallurgy, University of Cambridge, Cambridge, CB3 0FS, UK (e-mail: sch29@cam.ac.uk).

D. R. Vanden Bussche was with the Department of Materials Science and Metallurgy, University of Cambridge, Cambridge, CB3 OFS, U.K, and the Department of Inorganic and Physical Chemistry, Ghent University, Belgium.

A. Calleja, V. R. Vlad, and M. Vilardell are with OXOLUTIA SL, Campus de la UAB, Bellaterra, Barcelona, Spain.

X. Granados, T. Puig, and X. Obradors are with ICMAB-CSIC, Campus de la UAB, E-08193, Bellaterra, Barcelona, Spain

A. Usoskin is with Bruker HTS GmbH, Siemensstraße 88, 63755 Alzenau, Germany.

M. Soloviov, M. Vojenčiak, and F. Gömöry are with the Institute of Electrical Engineering, Slovak Academy of Sciences, Bratislava, Slovakia.

I. Van Driessche is with SCRIPTS, Department of Inorganic and Physical Chemistry, Ghent University, Belgium

M. Bäcker is with Deutsche Nanoschicht GmbH, Heisenbergstraße 16, D53359 Rheinbach, Germany

B. A. Glowacki is with the Department of Materials Science and Metallurgy, University of Cambridge, Cambridge, CB3 OFS, UK; the Department of Physics and Energy, University of Limerick, Castletroy, Ireland; and the Institute of Power Engineering, 02-981 Warsaw, Poland.

\section{INTRODUCTION}

$D_{\text {performance, the high cost of commercial }}^{\text {ESPITE GREAT improvements in critical current }}$ (RE) $\mathrm{Ba}_{2} \mathrm{Cu}_{3} \mathrm{O}_{7-\delta}$ coated conductors remains prohibitive, and $\mathrm{AC}$ losses further deter their selection for electric power applications. Hysteresis losses can be reduced by striation or etching, dividing the superconducting film into narrower filaments [1], but these subtractive processes further increase the production cost and complexity.

Inkjet printing methods combine the scalability and low cost typical of chemical solution deposition (CSD) routes with digital control and high resolution patterning, and hence have the potential to address both the cost and AC loss issues.

It has previously been demonstrated that superconducting $\mathrm{YBa}_{2} \mathrm{Cu}_{3} \mathrm{O}_{7-\delta}$ (YBCO) filaments can be deposited by inkjet printing on buffered Ni-W substrates, without subtractive processing steps, using TFA-based precursors [2], [3].

In the present work, YBCO filaments have been printed using low-fluorine precursor solutions on an alternative technically relevant substrate, stainless steel buffered with alternating beam assisted deposition (ABAD) YSZ.

Two alternative methods of preparing YBCO filaments were investigated. In the first method, 'direct printing', tracks of the YBCO precursor are printed directly on the buffered substrate. In the second, 'inverse' method previously reported by the present authors [3], oxide spacer tracks (e.g. $\left.\mathrm{CeO}_{2}\right)$ are first printed on the buffered substrate. A low-temperature heat treatment is then performed: the $\mathrm{CeO}_{2}$ template is not required to be highly textured. A full-width coating of the YBCO precursor is then printed, and separate YBCO filaments form between the $\mathrm{CeO}_{2}$ spacers during the subsequent heat treatment. The precursor deposited above the $\mathrm{CeO}_{2}$ spacers does not convert to superconducting YBCO.

The inverse printing approach is more complex, but it has some potential advantages. Firstly, the task of producing a thick superconducting layer (to maximize $I_{c}$ ) is separated from that of producing a narrow track. Secondly, the directly printed narrow tracks are no longer responsible for carrying current: the superconductor is deposited as a single continuous film. For directly printed YBCO tracks, a local defect, for example a defect on the substrate surface that prevents wetting, would result in a discontinuous YBCO filament. For the inverse approach, a spacer would be discontinuous at such a defect, but in many cases the current would be able to redistribute within the YBCO film. 
Scanning Hall probe characterization was used to demonstrate the successful formation of superconducting filaments in both samples. Finally, AC loss measurements were performed and compared to theoretical calculations.

\section{MATERIALS}

\section{A. Substrate}

Stainless steel tapes were coated with biaxially textured YSZ by alternating beam assisted deposition (ABAD) at Bruker HTS, and a $\sim 20-30 \mathrm{~nm}$ thick zirconium-doped ceria cap layer $\left(\mathrm{Ce}_{0.9} \mathrm{Zr}_{0.1} \mathrm{O}_{2}, \mathrm{CZO}\right)$ was added by spin coating in OXOLUTIA SL [4]. Short samples (30-100 mm long) of this SS/ABAD-YSZ/CZO substrate were ultrasonicated in ethanol for cleaning before printing.

\section{B. Precursor inks}

Low-fluorine YBCO precursor solutions were prepared in OXOLUTIA using a stoichiometric mixture of yttrium trifluoroacetate (Y-TFA), barium acetate (Ba-Ac) and copper acetate $(\mathrm{Cu}-\mathrm{Ac})$ in methanol and propionic acid, with the addition of triethanolamine (TEA), with a total metal ion concentration of $0.5 \mathrm{M}$ [4], [5].

For inverse printed filamentary $\mathrm{YBCO}, \mathrm{CeO}_{2}$ was chosen as the patterning material. The $\mathrm{CeO}_{2}$ precursor solution was prepared in Cambridge from cerium(III) acetate in propionic acid, with a cation concentration of $0.5 \mathrm{M}$ [6].

\section{METHODS}

\section{A. Inkjet printing}

Inkjet printing was performed using a bespoke positioning stage in a 'scanning head' configuration, with the printhead moving above a stationary substrate. The stage was designed, along with printing controllers and software developed in Cambridge, to permit the interchangeable use of different inkjet printing technologies.

TABLE I

INKJET PRINTING EQUIPMENT AND JETTING CHARACTERISTICS FOR YBCO AND CERIA TRACKS AND COATINGS

\begin{tabular}{|c|c|c|c|}
\hline & YBCO tracks & $\mathrm{CeO}_{2}$ tracks & YBCO films \\
\hline Inkjet printing device & $\begin{array}{c}\text { Microfab } \\
\text { MJ-ATP-01 }\end{array}$ & $\begin{array}{c}\text { Microfab } \\
\text { MJ-ATP-01 }\end{array}$ & $\begin{array}{c}\text { Gyger } \\
\text { SMLD } 300\end{array}$ \\
\hline Actuation technology & $\begin{array}{l}\text { Piezo- } \\
\text { electric }\end{array}$ & $\begin{array}{l}\text { Piezo- } \\
\text { electric }\end{array}$ & $\begin{array}{l}\text { Solenoid } \\
\text { valve }\end{array}$ \\
\hline Orifice diameter $(\mu \mathrm{m})$ & 60 & 60 & 100 \\
\hline Pressure (mbar) & -48 & -48 & 100 \\
\hline Jetting distance ${ }^{a}(\mathrm{~mm})$ & 1 & 1 & 4 \\
\hline Drop volume (pL) & 65 & 76 & 6000 \\
\hline Drop velocity $\left(\mathrm{m} \mathrm{s}^{-1}\right)$ & 1.2 & 1.4 & 2 \\
\hline
\end{tabular}

a Jetting distance is the typical distance of the orifice from the substrate

Two types of single-nozzle inkjet device were used in the present work. Tracks were printed using a MJ-ATP-01 microdispenser (Microfab Technologies Inc.). This device has a piezoelectric actuator and a $60 \mu \mathrm{m}$ orifice, typically producing drop volumes in the $50-100 \mathrm{pL}$ volume range. Continuous coatings, for which small volumes and high resolution were not required, were produced with an SMLD
300 micro valve (Fritz Gyger AG) with a $100 \mu \mathrm{m}$ orifice, typically producing drops with $5-15 \mathrm{~nL}$ volumes. Key parameters for the inks and printheads used in the present study are shown in Table I.

A strobed back-lit drop visualization system, with quantitative image analysis, was used to measure the drop volumes and velocities, as a guide to optimizing the printhead drive waveforms and pressure conditions.

\section{B. Thermal processing}

Heat treatments were performed in a furnace with a computer-controlled atmosphere.

For $\mathrm{CeO}_{2}$ tracks, heat treatment was performed for $1 \mathrm{~h}$ at $650{ }^{\circ} \mathrm{C}$ in Ar. This temperature was deliberately lower than the $1000{ }^{\circ} \mathrm{C}$ previously reported for $\mathrm{Gd}$-doped $\mathrm{CeO}_{2}$ coatings, as fully sintered well-textured tracks were not desired [6].

YBCO coatings and tracks were transferred to the furnace as soon as possible after deposition, and pyrolyzed with a slow ramp to $\sim 500{ }^{\circ} \mathrm{C}$ in a humid $\mathrm{O}_{2}$ atmosphere. After cooling, the conversion to $\mathrm{YBCO}\left(\sim 800^{\circ} \mathrm{C}, 200 \mathrm{ppm} \mathrm{O}_{2}\right)$ and oxygenation $\left(\sim 450^{\circ} \mathrm{C}, \mathrm{O}_{2}\right)$ were accomplished in a separate heat treatment.

\section{Scanning Hall probe magnetometry}

Samples were cooled in liquid nitrogen without applied magnetic field, and a field of $\sim 100 \mathrm{mT}$ was then applied for $20 \mathrm{~s}$. After a $300 \mathrm{~s}$ delay, a Hall probe (active area $\sim 0.1 \mathrm{~mm} \times 0.1 \mathrm{~mm}, 0.2 \mathrm{~mm}$ above the surface) was scanned over the sample and the magnetic field recorded at $0.2 \mathrm{~mm}$ intervals in the longitudinal and transverse directions.

\section{AC loss measurements}

AC magnetization losses were measured in an applied AC magnetic field by an established calibration free method [7]. Measurements were performed with applied field frequencies of 36, 72 and $144 \mathrm{~Hz}$, and the results compared with calculations for full-width and multi-filamentary samples using the Brandt-Indenbom model for a superconducting strip in perpendicular $\mathrm{AC}$ field [1].

\section{DiReCt PRINTING OF YBCO FILAMENTS}

The direct printing of YBCO filaments was performed using the piezoelectric micro-dispenser (Microfab MJ-ATP01). The drive waveform was first optimized based on drop visualization images, resulting in the formation of a single near-spherical drop with a volume of $65 \mathrm{pL}$ within $0.2 \mathrm{~mm}$ of the nozzle (Fig. 1). The typical drop velocity was $1.2 \mathrm{~m} \mathrm{~s}^{-1}$.

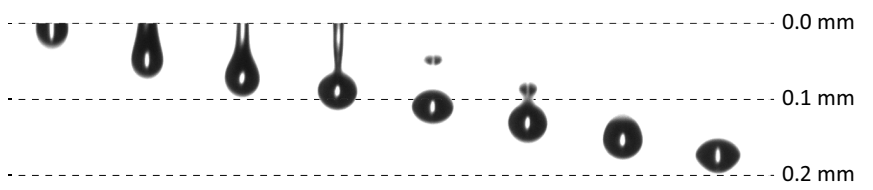

Fig. 1. Drop visualization images of the low-fluorine YBCO precursor ink during jetting from the Microfab MJ-ATP-01 piezoelectric dispenser. Images were obtained at $20 \mu$ s intervals and automated background removal processing applied.

A key challenge with directly-printed YBCO filaments is to increase the final YBCO thickness (out of plane) whilst retaining narrow tracks (in plane). The rheological properties 
of the ink must achieve a compromise between optimized wetting behavior on the substrate and optimized jetting behavior in the printhead; and there is generally found to be only a small range of drop separations for which uniform tracks are obtained. The YBCO thickness can then be increased either by increasing the ink concentration or by performing repeated printing passes. In the present study, the latter was investigated.

The sample shown in Fig. 2 was prepared with three filaments $\sim 2 \mathrm{~mm}$ wide. The first was printed in a single pass; to achieve a higher thickness, the second and third were formed from two and three printing passes respectively, leaving the sample to dry in air for $60 \mathrm{~s}$ between each pass.

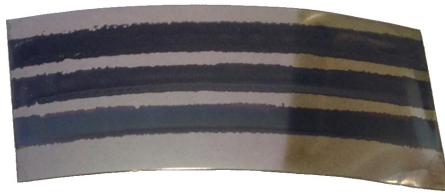

Fig. 2. Photograph of a sample with three directly-printed YBCO filaments, produced by 1,2 and 3 printing passes respectively to achieve different thicknesses. The substrate width is $12 \mathrm{~mm}$.

After heat treatment, the Hall probe scan (Fig. 3) revealed three distinct superconducting filaments, but the critical current performance evidently did not scale in proportion to the intended YBCO thickness. The low performance of the thickest filament (produced from three successive depositions) may be related to defects either introduced during pyrolysis and growth for the thicker film, or between and during successive printing stages, and highlights the need for optimization of all stages of the process for thick films.

The other two filaments, however, are rather uniform longitudinally, with $I_{c}$ peaking at $\sim 37 \mathrm{~A} / \mathrm{cm}$ width, which for the thinnest filament is a promising result. The key objective for future work is to retain this performance in thicker filamentary coatings.

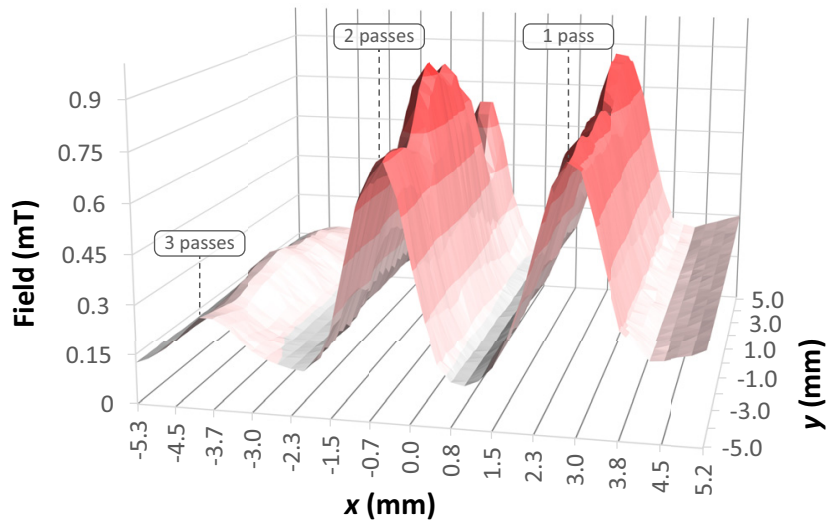

Fig. 3. Scanning Hall probe results for three directly-printed YBCO filaments of different thicknesses.

\section{INVERSE PRINTING OF YBCO FILAMENTS}

The inverse printing method used in the present study is shown schematically in Fig. 4.

$\mathrm{CeO}_{2}$ precursor tracks were printed with the piezoelectric micro-dispenser (Microfab MJ-ATP-01). The drive waveform was optimized in the same way as for the YBCO precursor ink, resulting in a single well-formed $76 \mathrm{pL}$ drop within $0.1 \mathrm{~mm}$ of the nozzle. $\mathrm{CeO}_{2}$ tracks are not very visible on metallic substrates; Fig. 5(a) shows an example from the authors' previous experiments on $\mathrm{Ni}-\mathrm{W}$ substrates.
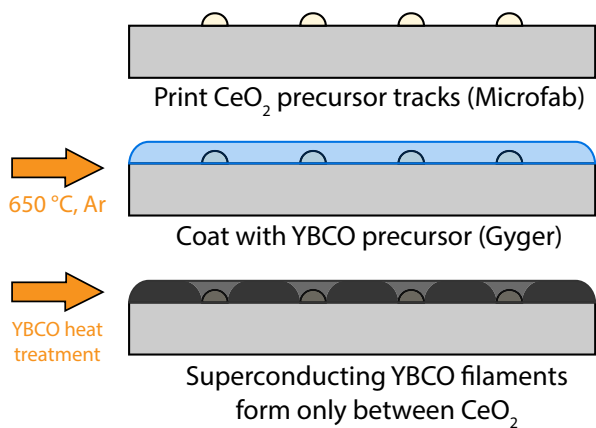

Fig. 4. Schematic illustration of the inverse printing route used in the present study.

After processing at $650{ }^{\circ} \mathrm{C}$ in $\mathrm{Ar}$, the presence of a (111) $\mathrm{CeO}_{2}$ peak in the X-ray diffraction pattern confirmed that poorly-textured polycrystalline $\mathrm{CeO}_{2}$ was formed, as intended.

A full-width coating of the YBCO precursor was then deposited, by printing a square array of drops with an SMLD 300 micro valve (see Table I), and the two-stage YBCO heat treatment applied. The resulting fully processed five-filament sample is shown in Fig. 5(b). The dark strips are the YBCO filaments that have grown between the $\mathrm{CeO}_{2}$ spacers.

(a)

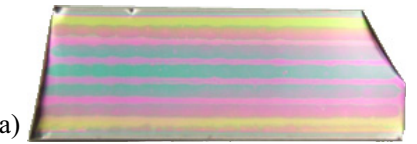

(b)

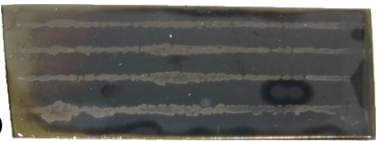

Fig. 5. Photographs of (a) $\mathrm{CeO}_{2}$ tracks printed on a buffered metallic substrate (in this case $10 \mathrm{~mm}$ wide $\mathrm{Ni}-\mathrm{W}$, from [3]), and (b) inverse printed YBCO filaments produced in the present study on $12 \mathrm{~mm}$ wide SS/ABADYSZ/CZO substrate.

The scanning Hall probe data in Fig. 6 confirm that five distinct superconducting filaments have been produced. It is clear that the performance of the edge filaments is poor, and that $I_{c}$ also decreases towards the ends of the sample. Edge effects are not uncommon in solution-processed coated conductors, often originating from the buffered substrate, and non-uniform wetting is also evident in the photograph.

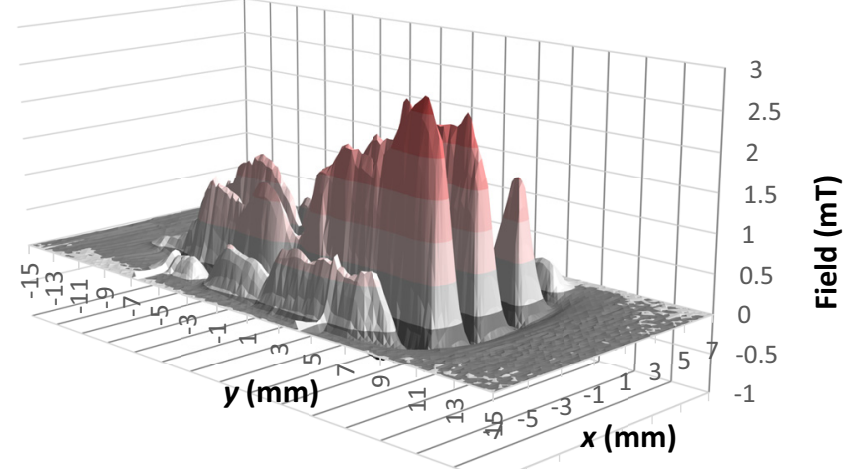

Fig. 6. Scanning Hall probe results for an inverse printed sample with five filaments.

The central filaments, however, have a very promising peak $I_{c}$ of $45 \mathrm{~A} / \mathrm{cm}$ width. The estimated YBCO thickness is $\sim 150 \mathrm{~nm}$, so this corresponds to a $J_{c}$ of $3 \mathrm{MA} \mathrm{cm}^{-2}$ at $77 \mathrm{~K}$, 
comparable to good full-width inkjet-printed YBCO films.

$\mathrm{AC}$ loss measurements on the same sample are shown in Fig. 7, with calculations from the Brandt-Indenbom model for comparison. The reduction in magnetization losses associated with division into five filaments is as expected. More detailed analysis of both hysteresis and coupling losses will require a longer and more uniform sample, which is planned for future work.

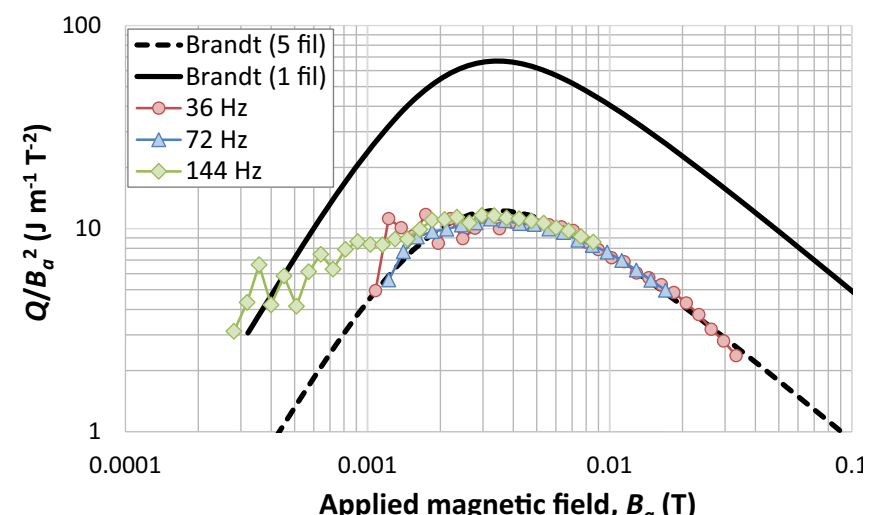

Fig. 7. Loss function for the inverse printed sample above, measured in an AC magnetic field at frequencies of 36,72 and $144 \mathrm{~Hz}$, compared with predictions for a full-width coating (' 1 fil') and for 5 filaments (' 5 fil').

\section{DISCUSSION}

The results above, taken alongside the authors' earlier study [3], demonstrate that multifilamentary YBCO structures can be deposited by inkjet printing on both RABiTS and ABAD derived buffered metallic substrates, using both the direct and inverse printing methods. Considering the low YBCO thickness of the test samples produced here, the peak $I_{c}$ values calculated from scanning Hall probe measurements for both techniques (37-45 A/cm width) are similar and promising.

The two printing routes each have distinct advantages and present their own challenges. One advantage of the direct printing approach is that, in principle, only a single deposition step is needed. The stable solution concentration is unlikely to far exceed double that used in the present study, which suggests that two or more deposition steps may still be needed to achieve sufficient thickness and $I_{c}$ in narrow tracks. If the lack of scaling of $I_{c}$ with multiple depositions found here is a general result, it would encourage the use of the inverse printing method. A priority for future work is therefore to establish the number of deposition passes which can be used with only low-temperature intermediate drying, and/or with intermediate pyrolysis.

The main limitation of the inverse-printed sample reported here is the lack of homogeneity, related to the sensitivity of chemical solution methods to the surface topology and condition of the substrate. For filaments printed to use the full width of the substrate, this is expected to apply to both direct and inverse printing. For the inverse printed sample, $I_{c}$ in the central filaments exceeded $70 \%$ of its peak value only over a continuous length of $7 \mathrm{~mm}$. These end effects have several contributions, but many - including substrate curvature, cutting artefacts, surface condition and air flow at the substrate edge - are related to the use of short substrate samples. Indeed, on $\sim 40 \mathrm{~mm}$ long samples not presented here, a uniform track with good uniformity over a length of $\sim 35 \mathrm{~mm}$ has been produced. In reel-to-reel production with a tensioned substrate, these issues would be further reduced.

The uniformity of individual YBCO filaments should also be better for samples produced by inverse printing, as shown schematically in Fig. 4. Directly printed tracks do not have a rectangular cross-section, and it has been observed that this variation in thickness hinders optimization of thermal processing, resulting in non-uniform superconducting properties [8]. For the inverse printing route, this nonuniformity applies only to the $\mathrm{CeO}_{2}$ spacers: the subsequently deposited YBCO film should have an almost flat surface over most of its width.

As mentioned in the introduction, the inverse printing method is also anticipated to be more reliable for long length production. For both direct and inverse printing, a major objective of ongoing research is to increase the sample length. As well as allowing a comparative test of defect tolerance, this is also required for transport $I_{c}$ measurements and a more thorough study of $\mathrm{AC}$ loss performance.

Finally, although the printing in this study was performed with single nozzle printing devices, it should be noted that the scalability of YBCO printing to production scale has been demonstrated using multi-nozzle printheads [9].

\section{CONCLUSION}

Multifilamentary YBCO has been successfully deposited by inkjet printing on SS/ABAD-YSZ/CZO substrates using a low-F YBCO precursor, both by direct piezoelectric printing, and by an inverse method using $\mathrm{CeO}_{2}$ spacers. Hall probe measurements identified distinct filaments with a peak $J_{c}$ of up to $3 \mathrm{MA} \mathrm{cm}^{-2}$, and the reduction in $\mathrm{AC}$ losses has been measured. Both approaches merit further investigation, but for directly printed filaments, a key challenge is to increase the YBCO thickness. For both approaches, substrate wetting must be optimized, and it remains to be demonstrated that continuous, non-coupled filaments can be produced in long lengths.

\section{REFERENCES}

[1] E. H. Brandt and M. Indenbom, "Type-II-superconductor strip with current in a perpendicular magnetic field", Phys. Rev. B, vol. 48, pp. 12893-12906, Nov. 1993.

[2] R. C. Duckworth, M. P. Paranthaman, M. S. Bhuiyan, F. A. List, and M. J. Gouge, IEEE Trans. Appl. Supercond., vol. 17, no. 2, pp. 3159-3162, Jun. 2007.

[3] S. C. Hopkins et al., "Inkjet printing of multifilamentary YBCO for low AC loss coated conductors", J. Phys. Conf. Ser., vol. 507, May 2014, Art. ID. 022010.

[4] E. Bartolomé et al., "Magnetic and structural characterization of inkjetprinted ${ }^{\mathrm{TFA}} \mathrm{YBa}_{2} \mathrm{Cu}_{3} \mathrm{O}_{7-\mathrm{x}} /{ }^{\mathrm{MOD}} \mathrm{CZO} /{ }^{\mathrm{ABAD}} \mathrm{YSZ} / \mathrm{SS}$ coated conductors", Supercond. Sci. Technol., vol. 26, Oct. 2013, Art. ID. 125004.

[5] N. Romà et al., "Preparation of anhydrous TFA solution for deposition of $\mathrm{YBa}_{2} \mathrm{Cu}_{3} \mathrm{O}_{7-\mathrm{x}}$ thin films", J. Phys. Conf. Ser., vol. 43, pp. 178-181, 2006.

[6] M. Mosiadz et al., "Inkjet printing of $\mathrm{Ce}_{0.8} \mathrm{Gd}_{0.2} \mathrm{O}_{2}$ thin films on Ni-5\%W flexible substrates", J. Sol-Gel Sci. Technol., vol. 54, no. 2, pp. 154-164, May 2010. 
[7] J. Souc, F. Gömöry and M. Vojenčiak, "Calibration free method for measurement of the AC magnetization loss", Supercond. Sci. Technol., vol. 18, pp. 592-595, Mar. 2005.

[8] C. Duckworth and M. Rupich, "Low AC Loss YBCO Coated Conductor Geometry by Direct Inkjet Printing", Am. Supercond. Corp., Devens, MA, USA, Tech. Rep. DOE/ER86300-1, Oct. 2009.

[9] I. Van Driessche et al., "Chemical solution deposition using ink-jet printing for YBCO coated conductors", Supercond. Sci. Technol., vol. 25, no. 6, Apr. 2012, Art. ID. 065017. 